\title{
THE COVID-19 PANDEMIC AND MACROECONOMIC FORECASTING: AN INTRODUCTION TO THE SPRING 2021 SPECIAL ISSUE
}

\author{
Ana Beatriz Galvao ${ }^{1,2,3,4 *}$ \\ ${ }^{1}$ Warwick Business School, University of Warwick, Coventry, United Kingdom \\ ${ }^{2}$ Fellow of the National Institute of Economic and Social Research, London, United Kingdom \\ ${ }^{3}$ Fellow of the Economic Statistics Centre of Excellence, London, United Kingdom \\ ${ }^{4}$ Fellow of the Centre for Economic Policy Research, London, United Kingdom \\ *Corresponding author. E-mail: Ana.Galvao@wbs.ac.uk
}

The Spring 2021 issue of the National Institute Economic Review examines the challenges of macroeconomic forecasting during the Covid-19 pandemic and publishes four solutions proposed by leading academic researchers in macroeconomic forecasting. ${ }^{.}$These contributions show how innovative methodologies can be applied to deal with the forecasting challenges thrown open by Covid-19. By organising this special issue, the National Institute Economic Review's editors hope to contribute to the debate on how to develop forecasting methods for economies subject to large shocks and instability.

To substantiate the observation that 2020 was a challenging year for forecasters, examine figure 1 , which shows quarterly GDP growth for the UK, the US and the Euro area for 2019 and 2020. It is a tale of two different worlds. In 2019, growth rates against the same quarter in the previous year (or year-on-year rates) were around 1 per cent for the Euro area and 2.3 per cent for the US, with UK values being in between. In 2020, the first quarter rate flipped to the negative side, but similar-sized declines have been observed in the past. Some extraordinary numbers were to follow. The decline in the UK GDP in 2020Q2 was 21 per cent. If we assume an average growth rate of 2 per cent a year, the observed 21 per cent fall implies that the UK economy generated the same value of products and services in 2020Q2 as in 2010Q2, 10 years earlier! By the end of 2020, the UK, the US and the Euro area economies were all looking better than in the second quarter, but still recording declines in GDP that were large enough to be classified as astonishing compared to the decades since World War II.

The question is then: how hard is it to predict the changes observed in 2020? Start with a straightforward forecasting rule: set the forecast for next-quarter GDP growth as the value observed for this quarter. Next, evaluate the forecasting performance using a quadratic loss function, that is, compute the square root of the average squared forecasting error (RMSFE) for a given period. If we calculate the RMSFE for the simple rule for UK GDP growth, as displayed in figure 1, we find a value of 0.29 for the four quarters of 2019, and 11.36 for the four quarters of 2020. Using this simple (but not necessarily inaccurate forecasting rule), we find that forecasting in 2020 is 40 times harder than it was in 2019 ! The challenging task of forecasting UK GDP growth in 2020 is also supported by the fan charts published by Hantzche and Young (2019) in November 2019 that attribute negligible probabilities for the growth rates actually observed in 2020 .

Castle et al. (2021) show that statistical forecasting models, which can adapt more quickly to these changes, perform better than well-specified structural models. They show that a well-specified model that links unemployment to costs and benefits of hiring fitted to data up to 2019 fails to predict the

\footnotetext{
${ }^{1}$ The papers were presented at an online workshop (via Zoom) on 20 November 2020. https://www.niesr.ac.uk/events/ impact-covid-19-pandemic-macroeconomic-forecasting.

(c) National Institute Economic Review, 2021.
} 


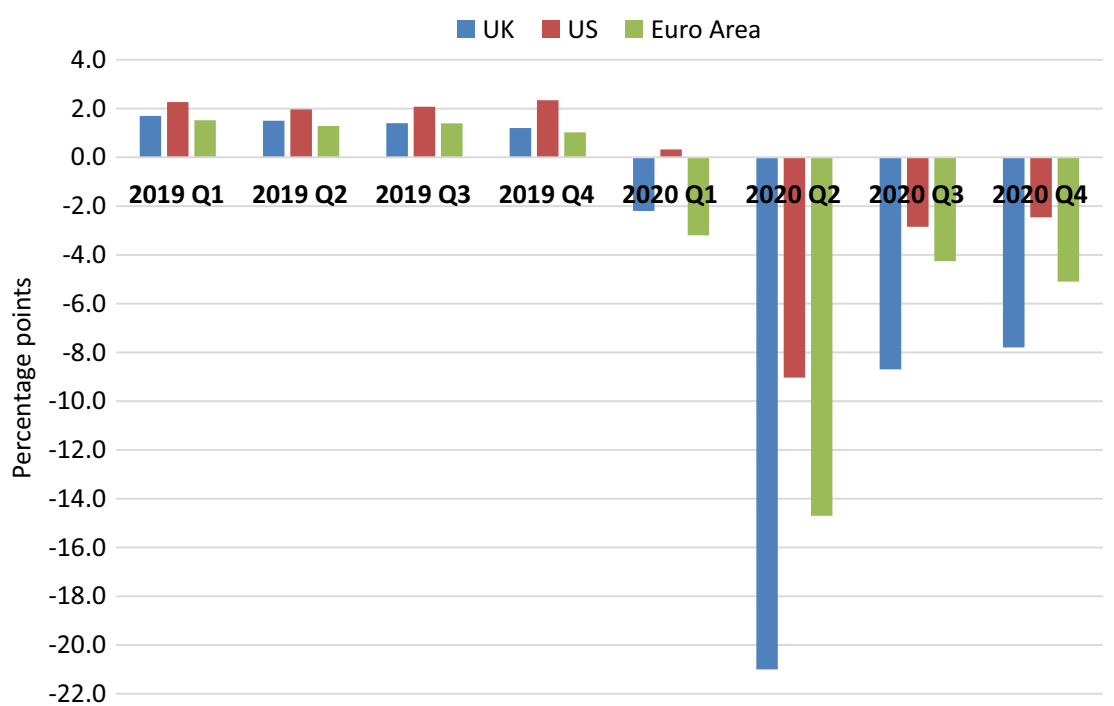

Figure 1. (Colour online) Year-on-year GDP growth for the UK, the US and the Euro area for the period 2019-2020

changes (or lack of) in the UK unemployment in 2020. An extrapolative statistical forecasting model performs better. They suggest that the performance gap between the statistical forecasting model and the structural forecasting model is a measure of the furlough scheme's impact on the UK unemployment rate.

In a similar line, Coulombe et al. (2021) provide additional evidence that adaptability is key in 2020 when forecasting UK monthly economic variables using a large set dataset of macro and financial variables. Nonlinear machine learning models (such as kernel ridge regressions and random forests) perform better than linear models (such as lasso and ridge regressions) that can deal with a large number of predictors, particularly for labour market variables. They provide evidence that innovative methods, such as autoregressive and the factor-augmented random forests, which use random forests to characterise time variation on the impact of past data on future values, perform better than traditional ones in terms of forecast accuracy.

Another critical issue is how to compute the expected uncertainty of forecasts during 2020. A simple and effective way to estimate GDP growth's predictive density starts by computing forecasting uncertainty using the observed past forecast errors of the forecasting method. Then, a Gaussian predicted density is computed using the point forecast and the estimated uncertainty. For the simple forecasting rule described earlier, we could use the RMSFE of the previous year as an estimate of forecasting uncertainty for the following year. It is clear that the 2019 estimate of 0.3 is too low for 2020, but the value of 11.4 for 2020 might be too large to characterise predictive densities for 2021. Koop et al. (2021) discuss how nowcasts for monthly UK GDP can be improved by considering modelling specifications that accommodate time variation in forecasting uncertainty. The authors review alternative approaches to accommodate this time variation able to deal with issues caused by the extraordinary forecasting errors observed for typical macroeconomic forecasting models. Using the available data at the time of writing (January 2021), however, it is still unclear how much of the observed forecasting failure in 2020 should be incorporated when measuring forecasting uncertainty and computing density forecasts for 2021 .

This special issue's contributions also show how macroeconomic forecasters have learned from, and contributed to, forecasting UK Covid-19 cases and deaths together with other health indicators such as hospital admissions and the Reproduction Rate (R). Harvey et al. (2021) and Castle et al. (2021) show how statistical time series models typically employed for macroeconomic forecasting can be adapted to forecast Covid-19 cases and deaths. Interestingly, the real-time data on Covid-19 cases suffer similar 
issues as GDP data: it is subject to data revisions. Harvey et al. (2021) consider a modelling approach to predict Covid-19 cases that combines Covid-19 cases by specimen and publication dates. Their approach also addresses issues of the ragged edge in the data, a significant problem when nowcasting GDP.

For an academic macroeconomic forecaster, 2020 was a fascinating year. We, as a profession, learned a lot from attempting to propose solutions to the challenges professional forecasters and policymakers faced. My view of the lessons learned from the contributions to this special issue is that adaptability is indeed key. Adaptability is not a characteristic of forecasting models that rely on how consumers and firms behave in normal times. Statistical models may then arise as a better choice than theoretically sound structural models. Some statistical model specifications, but not all, had an acceptable performance in 2020, as suggested by Castle et al. (2021) and Coulombe et al. (2021). Adaptability was also the essential characteristic of the forecasting methods presented to improve daily and weekly predictions of Covid-19 cases, the $R$ rate and deaths, as discussed by Harvey et al. (2021). Extreme adaptability, or overfitting, may, however, harm accuracy. Koop et al. (2021) suggest that models with no stochastic volatility may generate more robust density forecasts.

It remains to be seen whether the proposed forecasting methods may have a role in policy decisions. Many policymakers tend to prefer structural models to understand errors in terms of particular sectors and explore narratives about the economy. Many would maintain that forecasting is not simply about minimising forecast errors (Chadha, 2017). But by publishing these leading researchers' contributions using the most recent available data, we aim to disseminate the ongoing development of these innovative methods across professional forecasters. As better forecasts should lead to better policy decisions, we hope that these methodological advances will contribute to better policymaking.

\section{References}

Castle, J., Doornik, J. and Hendry, D. (2021), 'The value of robust statistical forecasts in the Covid-19 pandemic', National Institute Economic Review, forthcoming.

Chadha, J. (2017), 'Why forecast?', National Institute Economic Review, 239, pp. F4-F9. doi:10.1177/002795011723900103.

Coulombe, P.G., Marcellino, M. and Stevanovic, D. (2021), 'Can machine learning catch the Covid-19 recession?', National Institute Economic Review, forthcoming.

Hantzche, A. and Young, G. (2019), 'Prospects for the UK economy', National Institute Economic Review, 250, pp. F4-F9.

Harvey, A., Kattuman, P.A. and Thamotheram, C. (2021), 'Tracking the mutant: Forecasting and nowcasting Covid-19 in the UK in 2021', National Institute Economic Review, forthcoming.

Koop, G., McIntyre, S., Mitchell, J. and Poon, A. (2021), 'Nowcasting 'true' monthly US GDP during the pandemic', National Institute Economic Review, forthcoming.

Cite this article: Galvao, A. B. (2021), 'The Covid-19 pandemic and macroeconomic forecasting: An introduction to the Spring 2021 special issue', National Institute Economic Review, 256, pp. 16-18. https://doi.org/10.1017/nie.2021.11 